\title{
Correspondence
}

\section{Mammalian ncRNA-disease repository: a global view of ncRNA-mediated disease network}

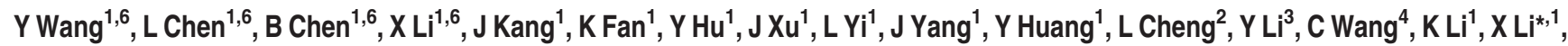 \\ $\mathrm{J} \mathrm{Xu}^{\star, 1,5}$ and $\mathrm{D}$ Wang ${ }^{\star, 1}$
}

Cell Death and Disease (2013) 4, e765; doi:10.1038/cddis.2013.292; published online 8 August 2013

Subject Category: Cancer

\section{Dear Editor}

Recently, substantial studies have begun to explore the functional diversity and mechanistic roles of ncRNAs in mammals. ${ }^{1}$ Now, it has become increasingly apparent that ncRNAs are involved in multiple major biological processes, such as developmental timing, fat metabolism and cell death. ${ }^{2}$ Furthermore, the epigenetic and genetic defects in ncRNAs and their processing machinery have been implicated in the etiology of many forms of diseases. ${ }^{3}$ Several databases that documented the relevance of the microRNAs(miRNAs) to diseases have been constructed and provided useful results. ${ }^{4,5}$ However, miRNAs are just the tip of the iceberg, other ncRNAs such as long non-coding RNAs (IncRNAs), PIWI-interacting RNAs (piRNAs) and small nucleolar RNAs (snoRNAs) have also been demonstrated to contribute to diseases. ${ }^{3,6}$ Accumulated evidence suggest the diverse noncoding RNAs (ncRNAs) involved in a wide variety of diseases progression. ${ }^{3,6,7}$ It is a key challenge for understanding the precise behavior of diverse ncRNAs in mammalian diseases and deciphering the cross-regulations among diseaseassociated ncRNAs. Because there was no repository focused on diverse ncRNA-disease relationships in mammals, we have developed a manually curated diverse ncRNA-disease repository (MNDR, www.rna-society.org/mndr/) by integrating evidence in three mammals. Totally, 807 IncRNA-associated, 229 miRNA-associated, 13 piRNA-associated and 100 snoRNA-associated entries for 1149 curated entries were documented for three mammals (866 Homo sapiensassociated, 251 Mus musculus-associated and 32 Rattus norvegicus-associated entries) (Table 1).

Recent investigations indicated there are complex regulations among diverse ncRNAs and protein-coding genes. Such as, PTEN gene and the PTEN pseudogenes (ptenp1, one of IncRNAs) share a high degree of sequence homology, changes in ptenp1 expression levels indirectly affect PTEN expression by sequestering PTEN-targeting miRNAs. ${ }^{8}$
Table 1 The statistics of the ncRNA-disease entries in MNDR database

\begin{tabular}{lccccc}
\hline Species & IncRNA & miRNA & piRNA & snoRNA & Total \\
\hline Homo sapiens & 753 & NA & 13 & 100 & 866 \\
Mus musculus & 50 & 201 & 0 & 0 & 251 \\
Rattus norvegicus & 4 & 28 & 0 & 0 & 32 \\
Total & 807 & 229 & 13 & 100 & 1149 \\
\hline
\end{tabular}

Thus understanding the mutual regulating pattern among diverse ncRNAs and protein-coding genes, particularly in disease conditions, is a key challenge. Thus, MNDR is not only a knowledge depository but providing us a good opportunity to view the ncRNA-mediated disease network globally (in visualization page: www.rna-society.org/mndr/visualization.html). Diverse ncRNAs and interaction genes were represented as nodes and the regulations were denoted as edges. Based on such a simplified ncRNA-mediated disease network, interesting observations have been achieved. The result showed that snoRNA $h t r$, as a hub node, has intensively linked to 21 interaction genes in the network. More important, through BCL2, BCL2L1 and BAX, the snoRNA htr can communicate with the IncRNA malat1 (Supplementary Figure 1). Another example is snoRNA $h t r$ and IncRNA $h 19$ are linked by E2F1 and MYC. When combined with human disease-associated miRNA evidence from mir2disease database, IncRNAs, miRNAs and snoRNAs, together with their interaction/target genes, can be integrated into bigger expanding ncRNA-mediated disease network. The biggest subnetwork has 129 nodes and 149 edges, involving 33 IncRNAs, 1 snoRNA, 19 miRNAs and 76 interaction protein-coding genes(Supplementary Figure 2). In this network, more regulations among diverse ncRNAs directly or indirectly via intermediate genes, IncRNA dgcr5, har1a and har1b, were connected with hsa-mir-21 via intermediate gene REST. Interestingly, hsa-mir-21 and snoRNA htr were linked by key anti-apoptosis gene $B C L 2$. Similar results were observed that

\footnotetext{
${ }^{1}$ College of Bioinformatics Science and Technology, Harbin Medical University, Harbin, China; ${ }^{2}$ State Key Laboratory of Emerging Infectious Diseases, Li Ka Shing Faculty of Medicine, The University of Hong Kong, Hong Kong SAR, China; ${ }^{3}$ Institute of Cardiovascular Sciences and Key Laboratory of Molecular Cardiovascular Sciences, Ministry of Education, Peking University Health Science Center, Beijing, China; ${ }^{4}$ Department of General Surgery, Huaihe Hospital of Henan University, Kaifeng, China and ${ }^{5}$ College of Bioengineering, Henan University of Technology, Zhengzhou, China

${ }^{*}$ Corresponding author: D Wang, J Xu and X Li, College of Bioinformatics Science and Technology, Harbin Medical University, Harbin 150081, China. Fax: 86 045186615922; E-mail: wangdong@ems.hrbmu.edu.cn (WD) or xujz0451@gmail.com (XJ) or lixia@ @rbmu.edu.cn (LX)

${ }^{6}$ These authors contributed equally to this work
} 
IncRNA dgcr5, har1a and har1b can also communicate with snoRNA htr through alternative route NFKB1-hsa-mir-9-REST. Hence, according to current data, the two pivot protein-coding genes (BCL2 and NFKB1) and several ncRNAs (IncRNA malat1, snoRNA htr and miRNA hsa-mir-21, has-mir-9) collectively play an important role in the ncRNA-mediated disease network (Supplementary Figure 2). Importantly, the crosstalk between IncRNA malat1 and miRNA hsa-mir-21 can be found conserved in mouse ncRNA-mediated disease network. Above observations indicated diverse ncRNAs could communicate with each other in disease state through some disease-associated genes in mammals, highlighting the complexity, conservative and plasticity of the regulatory relationships between diverse ncRNAs and protein-coding genes in diseases.

\section{Conflict of Interest}

The authors declare no conflict of interest.

Acknowledgements. This work was supported by the National Natural Science Foundation of China (31100901, 91129710, 61170154 and 61073136),
China Postdoctoral Science Foundation (2013M531064), Natural Science Foundation of Heilongjiang Province of China (QC2010012 and ZD201114), Heilongjiang Postdoctoral Foundation (LBH-Z12171), Henan University of Technology (2009BS040 and 11JCYJ11) and China Undergraduate Training Programs for Innovation (201210226016).

1. Frazer KA. Genome Res 2013; 22: 1599-1601.

2. Qureshi IA, Mehler MF. Nat Rev Neurosci 2012; 13: 528-541.

3. Esteller M. Nat Rev Genet 2011; 12: 861-874.

4. Jiang $Q$ et al. Nucleic Acids Res 2009; 37(Database issue): D98-D104.

5. Xu J, Li YH. Cell Death Differ 2012; 19: 1571.

6. Sana J et al. J Transl Med 2012; 10: 103.

7. Li L et al. Proc Natl Acad Sci USA 2009; 106: 12956-12961.

8. Poliseno L et al. Nature 2010; 465: 1033-1038.

Supplementary Information accompanies this paper on Cell Death and Disease website (http://www.nature.com/cddis) 